\title{
El cambio en las organizaciones del Estado
}

Marta Pérez Castaño*

* Profesora de la Facultad de Ciencias de la Administración de la Universidad del Valle. 



\section{EL NUEVO ROL DEL ESTADO COLOMBIANO}

No se debería hablar de cambio en el aparato público o de reforma de la administración pública, sin eludir el papel que se desea desempeñe el Estado frente a la sociedad.

Lastimosamente en Colombia, se planteó una política de redefinición delEstado, basada solamente en lo que se ha denominado la modernización del mismo, consistente en: a) reducción progresiva de la gestión estatal de aquellas actividades que puede cumplir más eficientemente el sector privado, b) promover la transición hacia un sistema de subsidios a los usuarios,c) eliminar regulaciones y trámites innecesarios y d) reestructurar las entidades.

Según el Plan Nacional de Desarrollo "La Revolución Pacífica" (1991), el reajuste estaba orientado a reducir el papel del Estado en la economía, bien sea eliminando su actividad productiva directa o abandonando la planeación indicativa y sectorial, la regulación directa, por la formulación de directrices generales y la definición de reglas de juego claras y estables para el fortalecimiento de las fuerzas del mercado, el estímulo a la competencia y un mayor peso de las decisiones privadas en la determinación del ritmo y dirección de la economía.

AI respecto, Lechner (1993) plantea que el debate dado sobre la modernización del Estado se ha hecho con discusiones compartimentalizadas, con un enfoque pragmático, demasiado administrativo, que no pone en discusión el concepto mismo de Estado y por lo tanto nada puede decirse acerca del Estado que se desea construir.

Está demostrado que la problemática del Estado en Latinoamérica no se puede reducir al debate del tamaño del Estado, ya que cuando comparamos la relación entre el volumen de personal empleado en el sector público en su conjunto y el tamaño de la población, los resultados no son diferentes a la misma relación en países desarrollados. Sobre lo que no debería haber ninguna duda, es, acerca de la capacidad y calidad de su gestión. "Reducir el aparato del Estado simplemente es escapar al 
problema, pues en lo que queda se seguirá evidenciando posiblemente el anquilosamiento" (Cabrero y Escotto, 1992).

Otro elemento que permanece en la discusión sobre el rol del Estado, es el carácter y alcance de su intervención; al respecto coinciden diversos autores que éste es un problema de principios y de hecho el Estado está obligado a intervenir para garantizar la democracia y la equidad social. Es más, como bien plantea Weber: "Ningún país, ni Alemania, Francia, Japón, Estados Unidos, etc., pudo desarrollarse sin la acción central del Estado para crear una burguesía nacional capitalista". Lo que sí preocupa es la capacidad real que tiene el Estado en Colombia para realizar una intervención efectiva.

No se puede reducir la problemática de la Gestión del Estado a su "modernización", entendiendo por ésta como lo nuevo, como la simple incorporación de nuevas tecnologías administrativas, como un fin en sí mismo y no comoun medio. No es entonces suficiente este planteamiento, debería concebirse más bien como la necesidad de una verdadera transformación del Estado, donde la modernización será uno de los aspectos a tratar.

Según Malaver (1993), en Colombia "Las aspiraciones sociales rebasan la modernización del Estado y más bien ésta debería posibilitar y orientar el tránsito del país hacia la reconversión económica y la modernidad sociocultural. De materializar la Constitución las aspiraciones que ella encarna, el país transitará a la modernidad. Hacia allí apunta la búsqueda de una sociedad más abierta, pluralista y participativa, los principios de soberanía popular; la prevalencia de la solidaridad y el interés general propios de un Estado social de derecho; la defensa del medio ambiente; el reconocimiento de una diversidad étnica y cultural, la descentralización y la participación democrática mediante figuras comola elección popular, veedurías y fiscalización de la comunidad o instituciones como la defensoría del pueblo o la tutela. Todo lo anterior tiende a reformular las formas de relación Estado sociedad civil".

Encarar la situación económica y la necesidad de democratización exigen un replanteamiento de fondo sobre las relaciones del Estado con 
la ciudadanía, exige "Transformaciones de fondo en el aparato público y una elevación sustancial de su capacidad de gestión... Existe en Latinoamérica una pronunciada brecha entre la capacidad administrativa delEstadoy las responsabilidades crecientes que le han sido encomendadas en las últimas décadas. Sin capacidad de gestión estatal, las políticas y proyectos económicos y sociales más consistentes, tropezarán con un cuello de botella que reducirá drásticamente sus posibilidades de cumplimiento efectivo. Sin reformar la actual maquinaria del Estado, dotándola de potencialidades y mecanismos administrativos necesarios, el sistema democrático no operará como tal en aspectos sustanciales, impidiendo los consensos y debilitando la legitimidad" (Kilksberg, 1989).

\section{LA NECESIDAD DE TRANSFORMAR EL ESTADO}

Se han venido llevando a cabo en Latinoamérica diversos tipos de reformas en el Estado que bien vale la pena tipificar según su alcance y orientación, con el objeto de comprender y reconocer las características de la propuesta de Modernización del Estado en Colombia. De igual manera debe tenerse presente qué le implica al Estado afrontar las exigencias de desarrollo social y económico y la democratización.

\section{ORIENTACION Y PROFUNDIDAD DEL CAMBIO}

Cabrero y Escotto (1992) parten del análisis de diversas experiencias de Reforma en América Latina y conceptualizan las situaciones de cambio que pueden presentarse caracterizándolas como: reestructuración. reforma administrativa, renovación institucional y lo que ellos denominan propiamente reforma estatal. En el cuadro Situaciones de Cambio, se puede observar la descripción de cada una de estas categorías.

Consideran los autores que la confluencia simultánea de procesos de democratización, modernización económica y reorientación estatal, influyen para hacer más complejo el mecanismo de transformación de las organizaciones del Estado y que una situación de cambio podría entonces entenderse como el marco de premisas y supuestos en cuyo interior se pretende inducir el proceso. Este marco está dado por la definición de dos 
características:

- La orientación: los ámbitos de acción y límites planteados, si se pretende un cambio sólo al interior del aparato o bien que incluya también la relación del aparato con los grupos de la sociedad.

- La profundidad con la que se pretende inducir dicho cambio.

SITUACIONES DE CAMBIO

\begin{tabular}{|c|c|c|c|}
\hline & & \multicolumn{2}{|c|}{ ORIENTACION DEL CAMBIO } \\
\hline & & Intraorganizacional & $\begin{array}{c}\text { Relación } \\
\text { Organización-Contexto }\end{array}$ \\
\hline \multirow{2}{*}{ 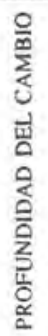 } & $\begin{array}{l}\text { Focalización sobre } \\
\text { estructuras organi- } \\
\text { zacionales }\end{array}$ & Reestructuración & $\begin{array}{c}\text { Reforma } \\
\text { Administrativa }\end{array}$ \\
\hline & $\begin{array}{l}\text { Focalización sobre } \\
\text { estructurgscompor- } \\
\text { tamientos organiza- } \\
\text { cionales }\end{array}$ & $\begin{array}{l}\text { Renovación } \\
\text { Institucional }\end{array}$ & $\begin{array}{l}\text { Reforma } \\
\text { Estatal }\end{array}$ \\
\hline
\end{tabular}

Las situaciones de cambio planteadas por los autores son las siguientes:

La Reestructuración del aparato, caracterizada por acciones relacionadas con: a) redimensionamiento del aparato, b) cambios en las funciones, procedimientos y normatividad y c) nuevos diseños estructurales, "no modifican necesariamente la estructura de poder ni la dinámica de los procesos decisorios. Se trata de una estrategia conducida por la propia cúpula del aparato y la pirámide suele adaptarse a la nueva configuración".

La Reforma administrativa, en la cual se acepta la necesidad de una readecuación del aparato no sólo a las nuevas condiciones económicas sino a las nuevas necesidades de la sociedad, mayor cobertura de acción y agilidad de las demandas y servicios. La preocupación fundamental es buscar una rearticulación con los usuarios, es decir, parte de la actividad de las organizaciones se orienta al contexto, dejan de funcionar íntegramente para sí mismas. "La conducción del cambio continúa 
siendo desde la cúpula organizacional y no garantiza un proceso profundo de transformación, en ocasiones se asume una 'nueva imagen' para con el público y no necesariamente un nuevo tipo de relación". En esta situación "las formas han cambiado pero los vicios prevalecen"; se ha supuesto que las organizaciones públicas son maquinarias que con una nueva tecnología administrativa pueden modificarse.

Las acciones que sustentan esta situación son las siguientes: a) procesos de simplificación administrativa, b) desregulación, c) modernización de organismos y dependencias y d) cambios en funciones y normatividad.

La Renovación institucional intenta modificar los comportamientos, valores y actitudes de los integrantes de las organizaciones públicas. Se acepta que la transformación se dará en la medida en que se pueda llegar a modificaciones en la estructura de poder, en la forma y orientación de las decisiones, el compromiso en la acción y en la identificación con la función pública. La complejidad del proceso es mucho mayor porque el reto consiste en inducir un cambio organizacional profundo; además "es un proceso que si bien se induce de arriba hacia abajo, posteriormente adquiere una dinámica propia de reacomodos y desencadena procesos de negociación y acuerdos internos que con frecuencia generan conflictos organizacionales". En el corto plazo se asumen costos de energía, en el largo plazo se genera una dinámica de acción organizacional orientada al servicio público como valor institucional.

Las acciones que se realizan son las siguientes : a) profesionalización del servicio público, b) procesos de identidad institucional, c) generación de un nuevo esquema de compensaciones y contribuciones institucionales y d) mecanísmos de ascenso jerárquico asociado a logros profesionales.

La Reforma estatal, surge de una crisis de legitimidad del aparato; al aceptar esta situación como premisa, el proceso de cambio forma parte de unaredefinición del papeldel Estado. Bajoestepanorama las organizaciones públicas pasan a ser los mecanismos de ajuste e interlocución entre el Estado y la sociedad civil. Implica organizaciones con capacidad de interacción, negociación y mantenimiento de una calidad y armonía en relación con los agentes de la sociedad. Requiere renovación en estructuras y comportamientos en el interior. Se asume un replan- 
teamiento global de los fines y medios de las organizaciones públicas.

El Gobierno de turno tiene que asumir costos altos cuando se embarca en una reforma de este tipo:

- El reconocimiento autocrítico de un deterioro o agotamiento del aparato institucional del Estado

- La apertura a la participación permanente de grupos sociales y agentes no gubernamentales en sus acciones

- Aceptación del riesgo de perder en cierto momento el control del proceso de cambio.

Las acciones que se requieren para lograr este tipo de cambio son: a) descentralización ejecutada a profundidad, b) ceder espacios de decisión a otras instituciones, c) modificar estructuras de poder internas y d) asumir el papel como instituciones de servicio y no de dominación.

Consideran los autores que la tibieza de las reformas en algunos países se debe a:

- Inseguridad del gobierno en turno en emprender un proceso de cambio profundo que se le salga de control, sino que pueda desbordarlo en caso de si evoluciona como detonador de una crisis política.

- Incapacidad para inducir cambios profundos en las organizaciones públicas, las cuales se caracterizan por una inercia heredada de una fase del Estado omnipresente y unilateral que las llevó a aislarse de la sociedad y a perpetuar juegos de poder internos ajenos a su función primordial.

\section{EXIGENCIAS DEL CAMBIO}

$\mathrm{Al}$ asumir el Estado responsabilidades de diversa índole con la sociedad civil, de una parte debe estar en capacidad de afrontar las exigencias del desarrollo económico y social del municipio, región y nación, y de otra parte, tener capacidad para afrontar los requerimientos de la democratización de acuerdo al mandato constitucional.

En el cuadro siguiente se resumen los planteamientos que hace Bernardo Kliksberg (1989) sobre las implicaciones del cambio del aparato público para darle vía efectivamente a tales exigencias. 
Para AFrontar LAS EXIGENCIAS DEL DESARROLLO SOCIAL Y ECONÓMICO

- Productivizar el gasto público.

- Articulación sustantiva del aparato estatal.

- Variar profundamente la imagen del servicio público.

- Estímulo decidido a la creatividad organizacional.

- Desarrollo pleno de los recursos humanos del sector público.

- Desarrollar capacidades para gerenciar la complejidad.

- Reconstrucción de los sistemas de información.

- Transformaciones estructurales en la gestión de empresas públicas.

- Desarrollar capacidades para manejar programas sociales masivos.

- Desarrollar capacidades para implementar programas multiinstitucionales.

- Establecer mecanismos efectivos de evaluación.

- Producción orgánica de posibilidades productivas.

- Desarrollar capacidades para la integración y la integración regional.

- Aprovechamiento pleno de las potencialidades del poder de negociación regional.
PARA AFRONTAR LAS EXIGENCIAS DE LA DEMOCRATIZACIÓN

- Afianzamiento de la autodeterminación y soberanía nacional.

- Dar participación y representatividad a los diversos grupos sociales.

- Estimular la participación ciudadana en la gestión pública.

- Posibilitar la real fluidez del juego democrático, ser transparente en sus decisiones.

- Ser democrático al interior del aparato público.

- Erradicar la arbitrariedad burocrática, respetar los derechos de los ciudadanos y su igualdad.

- Encontrar alternativas de acción para terminar con la corrupción administrativa.

- Impulsar las potencialidades de organización social de la sociedad civil. 


\section{LA CAPACIDAD DE GESTION DEL ESTADO}

De los nuevos paradigmas en la Reforma del Estado se deben derivar algunos principios fundamentales para enmarcar el cómo debe hacerse un diagnóstico de la capacidad de gestión pública yen particular de organismos que tienen la responsabilidad de gobernar. Se entiende que desarrollar la capacidad de la gestión pública, es tener capacidad organizativa para maximizar la riqueza de la sociedad, para prestar servicios que garanticen un determinado nivel de bienestar social y económico, y regular comportamientos individuales y colectivos.

Si bien es cierto que la debilidad institucional del gobierno tiene origen en su situación administrativa y gerencial, como lo hemos observado en los nuevos paradigmas de la Reforma del Estado, no se puede reducir el análisis y diagnóstico de su capacidad de gestión a la problemática administrativa. Es necesario comprender que existe una dimensión de naturaleza política, asociada a la capacidad para gobernar. Como plantea Motta, problemas que en verdad corresponden a temas de participación política, descentralización y transferencia de poder, son tratados comúnmente, como si fueran de coordinación y control y la simple implementación de nuevos mecanismos administrativos no garantiza la eficacia de las soluciones.

La problemática institucional se entiende mejor, cuando se parte del análisis de las funciones del Estado con la sociedad y es esta relación la que debe cambiar en una perspectiva democrática.

La legitimidad en la administración pública no solo depende de la racionalidad administrativa, sino del compromiso del gobierno con el desarrollo social y económico, de sus nuevas relaciones con los gobernados.

Gobernar significa tomar decisiones sobre alternativas de acción para la sociedad. Tales alternativas se basan, en principio, en el interés público expresado colectivamente, de acuerdo con un proceso administrativo donde demandas y apoyos son convertidos en normas, productos y servicios. De ahí resulta una distribución de derechos y deberes, beneficios y costos, fundamentados en la autoridad (Matus, 1993). 
Por lo tanto, se entiende que cuando se habla de un programa de desarrollo institucional, para elevar la capacidad de gestión de la administración municipal, se hace necesario tener conciencia que nos estamos refiriendo a una problemática que va mucho más allá de la dimensión administrativa de la gestión, como se entiende comúnmente.

Al respecto plantea Matus (1993) que elevar la capacidad de la gestión del gobierno supone "reformar y quizá revolucionar las reglas de responsabilidad y gobernabilidad del aparato público", lo cual a su vez implica "una estrategia para vencer la sólida inercia de los sistemas centralizados de baja responsabilidad".

\section{EL DESEMPEÑO DE LAS ORGANIZACIONES}

\section{UN CAMBIO DE CULTURA ORGANIZATIVA}

Según Matus (1993), en las organizaciones se establece una relación dinámica entre su cultura, sus prácticas de trabajo y sus formas organizativas, que se puede visualizar a través del triángulo mostrado en el cuadro siguiente:

\section{EL DESEMPEÑO DE LAS ORGANIZACIONES}

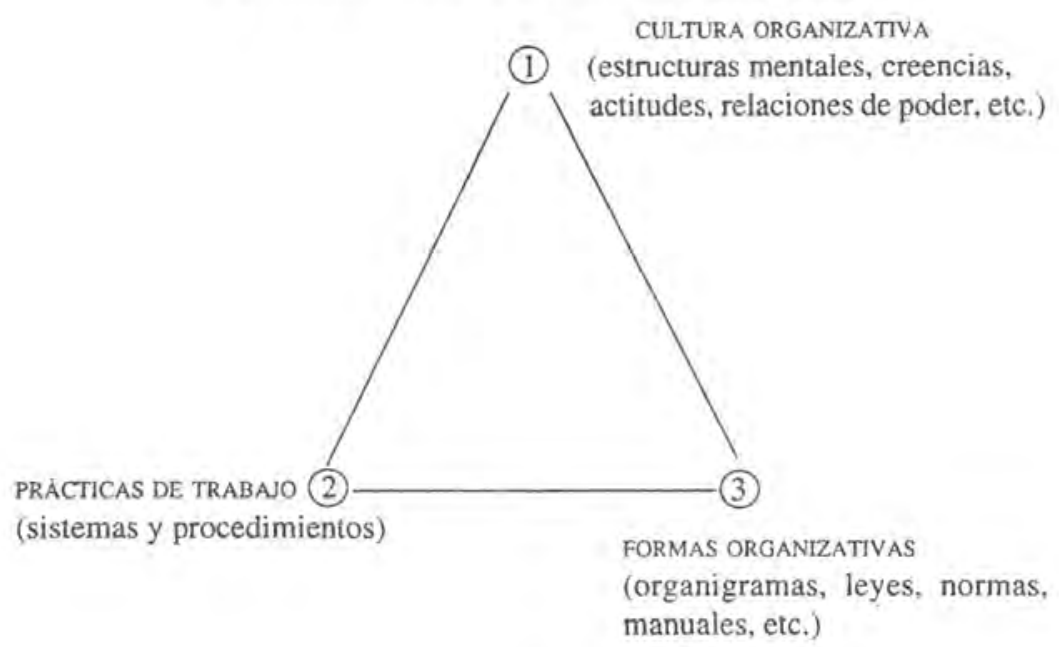

CUAdernos de administración No 22/UNIVERSIDAd deL VAlle/MAyo de 1996 
De las prácticas de trabajo establecidas en la organización, depende la calidad de la gestión pública, sus resultados. De hecho, la eficiencia y la eficacia de la organización están íntimamente ligadas con la orientación y calidad de las prácticas de trabajo.

Las formas organizativas (leyes, normas, organigramas, manuales, etc.), condicionan las prácticas de trabajo, mas no las cambia. Un nuevo organigrama no crea nuevas prácticas de trabajo.

Solamente la cultura organizacional que toca con las estructuras mentales, puede modificar las prácticas de trabajo. Se entiende por cultura organizativa, las ideas, creencias, actitudes, intereses, valores, símbolos, hábitos y ritos que caracterizan una organización.

No se debería iniciar un cambio en la gestión, sustentado en las formas organizativas o en las prácticas de trabajo. La organización real se impone sobre la organización formal. El proceso causal que rige el cambio es el siguiente: estructuras mentales $\rightarrow$ prácticas de trabajo $\rightarrow$ formas organizativas.

Un cambio de la cultura institucional, de las estructuras mentales, supone desarrollar capacidad para el autoanálisis y por supuesto, la autocrítica, supone también capacidad para asimilar teorías e interés por entrenarse en nuevos procesos.

Las prácticas de trabajo se cambian con sistemas y métodos, apoyados con capacitación, entrenamiento, asesoría y asistencia técnica.

Las formas organizativas se cambian con decisiones formales.

Los dirigentes forjan valores que aglutinan a la organización en pro de su desarrollo y su futuro. La profesionalización del servicio público, la identificación con los objetivos que se propone una administración gubernamental, se constituyen en elementos determinantes del desempeño de la organización.

Desarrollar la capacidad de gestión de la administración municipal, supone entonces, transformar su organización, lo cual implica una ruptura con los comportamientos y actitudes existentes, esta ruptura debe ser gradual. Motta considera que debe verse como un proceso acumulativo y permanente, que "progresa en medio de discontinuidades, conflictos y tensiones que no se resuelven en el corto plazo, ni en momentos predefinidos" 
Esto implica, como bien lo plantea Kliksberg, sustituir el enfoque prescriptivo por el heurístico: no someter la realidad a una normativa; explorar, experimentar y trabajar en marcos amplios de incertidumbre. Implica desarrollar capacidad para innovar en la gestión pública, de encontrar creativamente soluciones a los problemas detectados, para vencer las rigideces estructurales y la resistencia al cambio.

\section{LA INNOVACION EN LA GESTION PÚBLICA}

Se requiere desarrollar la capacidad de adaptación a los cambios, teniendo presente la turbulencia e incertidumbre del medio ambiente actual. No se puede proyectar el futuro de la organización con base en el pasado, no necesariamente lo que dio resultados en el pasado, los garantiza para el futuro.

La organización innova cuando se toma la decisión de reformular sus valores, sus prácticas de trabajo y sus formas organizativas y adaptarse al momento histórico. La decisión de innovar, de cambiar exige entonces, además de la voluntad de poner en marcha ideas nuevas, un conocimiento del contexto organizacional en el cual se propone efectuar el cambio.

La transición de lo antiguo a lo nuevo, no se puede ver con una lógica lineal ni secuencial, se trata de una ruptura y como tal es conflictiva, agudiza las contradicciones entre las fuerzas que buscan la estabilidad, $e$ l statu quo organizacional, y las que promueven el cambio.

Desarrollar capacidades para adaptarse a los cambios implica pensar en organizaciones flexibles, capaces de reestructurarse cuando se requiera.

\section{LA ESTRUCTURA ORGÁNICA QUE SE REQUIERE}

Con el objeto de lograr una efectiva descentralización al interior de la organización gubernamental y poder responder con rapidez a los cambios del contexto, inclusive actuar proactivamente, se requiere cambiar las estructuras organizativas actuales por aquellas que tengan las siguientes características:

Estructuras planas, en las cuales se reducen significativamente los niveles jerárquicos, promoviendo así las relaciones horizontales. 
Estructuras en las cuales se refleje la importancia y el poder de decisión que requieren los procesos centrales de la administración gubernamental, los programas y proyectos en que se ha comprometido el gobernante.

Estructuras que propicien y faciliten el contacto y el servicio a la ciudadanía.

Con unidades orgánicas y funcionarios que respondan por los programas y proyectos, lo cual implica rendición de cuentas y evaluación de desempeño sobre los compromisos adquiridos.

Con unidades orgánicas que tengan autonomía e independencia, es decir, que tengan poder de decisión sobre el manejo de los recursos: sobre la vinculación de personal, sobre la elaboración y ejecución del presupuesto, sobre la compra de equipos y materiales, sobre la contratación de obras y asesorías, etc.

Estructuras que propicien el trabajo de equipo, el trabajo interdisciplinario.

Con competencias y responsabilidades establecidas frente a la relación con la sociedad civil en general, y en particular, en relación con organizaciones formales e informales de usuarios de servicios y ciudadanos.

\section{SUPERAR LA DICOTOMIA POLITICA Y ADMINISTRACION}

La naturaleza de la administración pública incluye la dinámica política y por tanto el comportamiento de la organización se hace más eficiente cuando se supera el divorcio existente entre política y administración, como se ha demostrado en investigaciones realizadas por Crozier.

No se puede seguir pensando que existe una dirección política elegida por la población, que dicta las órdenes y un aparato que las ejecuta. Si gobernar implica tomar decisiones, debe darse una interrelación efectiva entre el análisis técnico y el político y dotar a la administración de métodos y mecanismos de gestión que permita asumirlo así. 


\section{LA CAPACIDAD PARA PROMOVER EL DESARROLLO DE ACTIVIDADES PRODUCTIVAS}

Como bien loplantea Buller, un gobierno considerado en su integralidad de Estado/sociedad civil ha de ser capaz de reconocer, estimular y encauzar el potencial económico de su comunidad. Esto implica que el gobernante y su administración desarrollen capacidades para identificar oportunidades de inversión que favorezcan el fortalecimiento y creación de proyectos productivos; igualmente se debe convertir en una importante tarea promover y atraer la inversión privada interna y externa en el municipio y la región.

Le exige esto a la administración pública, además de tener visión del contex to que vaya más allá de su ámbito, que lo inscriba en las posibilidades regionales, tener un sistema de planeación estratégica que permanentemente le dé elementos para reconocer y evaluar las nuevas oportunidades.

\section{LA GERENCIA DE POLITICAS}

La gerencia no administra solamente recursos, administra políticas, lo cual por supuesto implica tener una óptica de mayor alcance que la administración del presupuesto del presente y siguiente año como ha sido la tradición. "El papel principal de la gerencia pública en nuestros tiempos es el vinculado con las políticas" (Kliksberg).

\section{EL DESARROLLO DE LOS RECURSOS MUMANOS}

La capacidad de gestión de una organización depende directamente de las aptitudes de quienes la conforman. Es por ello, que un programa de desarrollo institucional debe incidir en el cambio de la administración de personal. Partiendo de la selección que debería hacerse evaluando los perfiles adecuados a las responsabilidades que se van a asumir lo cual implica implantar sistemas apropiados.

Sistemáticamente la administración debe propiciar el desarrollo de las potencialidades de su personal, esto exige invertir en capacitación y 
entrenamiento y disponer la organización para que esto pueda tener prioridad y viabilidad.

\section{BIBLIOGRAFIA}

Buller, EduARDo, "El desarrollo institucional municipal como estrategia de intervención para el cambio y como estrategia de desarrollo local", Cuaderno de Desarrollo Local.

Cabrero, Enrique y Escotto, Teresita, "Evolución reciente de los procesos de Reforma de la Administración Pública y su efecto en los modelos organizacionales", CIDE, México, 1992.

KuiksBerg, Bernardo, "¿Cómo transformar al Estado? Más allá de mitos y dogmas", Fondo de Cultura Económica, México, 1989.

"Nuevas fronteras en materia de gerencia en América Latina", Revista de la CEPAL NO. 31.

LECHNER, NORBERT, "Apuntes sobre las transformaciones del Estado", Bogotá, Revista FORO, No.21, 1993

MALAVER, FloRentino, "La formación postuniversitaria en gestión pública y la modernización del Estado: elementos para una evaluación de su calidad y perspectivas", Mimeo, Bogotá, agosto de 1993.

Matus, CARLOS, "Sobre lateoría de las macroorganizaciones", Mimeo, Fundación Altadir, Caracas, 1993.

MotTa, Paulo Roberto, "Innovación y Democratización de la Gestión Pública", Documento del Centro Latinoamericano de Administración parael Desarrollo.

Presidencia de la República, Consejería para la modernización del Estado, "Hacia un Estado Moderno", Bogotá, febrero de 1993.

Presidencia de la República, DNP, "La Revolución Pacífica", Bogotá, 1991. 